\title{
Management of haemorrhage secondary to a pseudoaneurysm of the gastroduodenal artery
}

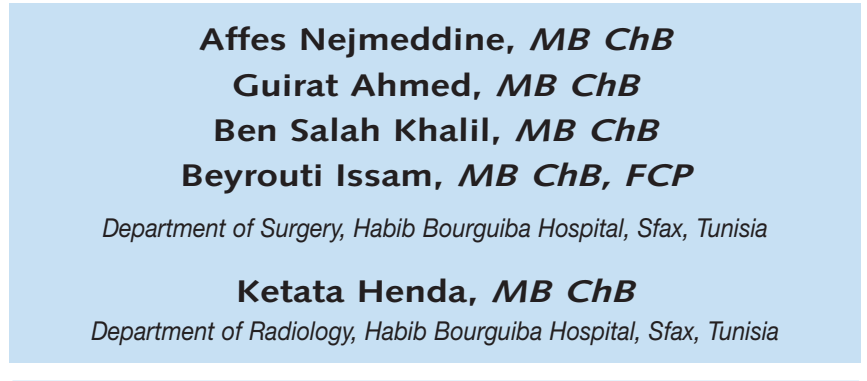

\section{Abstract}

We report the case of a 31-year-old man with chronic pancreatitis and haematemesis secondary to a gastroduodenal artery pseudoaneurysm. No evidence-based guidelines exist regarding the optimal treatment modality for this condition. In referring to this case, we emphasise the value of coeliac arteriography for confirmation and transcatheter embolisation.

\section{Introduction}

Gastroduodenal artery pseudoaneurysms are rare. They mainly develop secondary to pancreatitis. They are reported to occur in $10 \%$ of chronic pancreatitis cases, with a high mortality rate after rupture. The optimal treatment of arterial pseudoaneurysms following pancreatitis is controversial, with operative and interventional methods as options.

\section{Case report}

A 31-year-old man with a history of alcohol abuse was admitted to our hospital with recurrent episodes of epigastric pain, haematemesis and melena. He reported a weight loss of $13 \mathrm{~kg}$ during the preceding month.

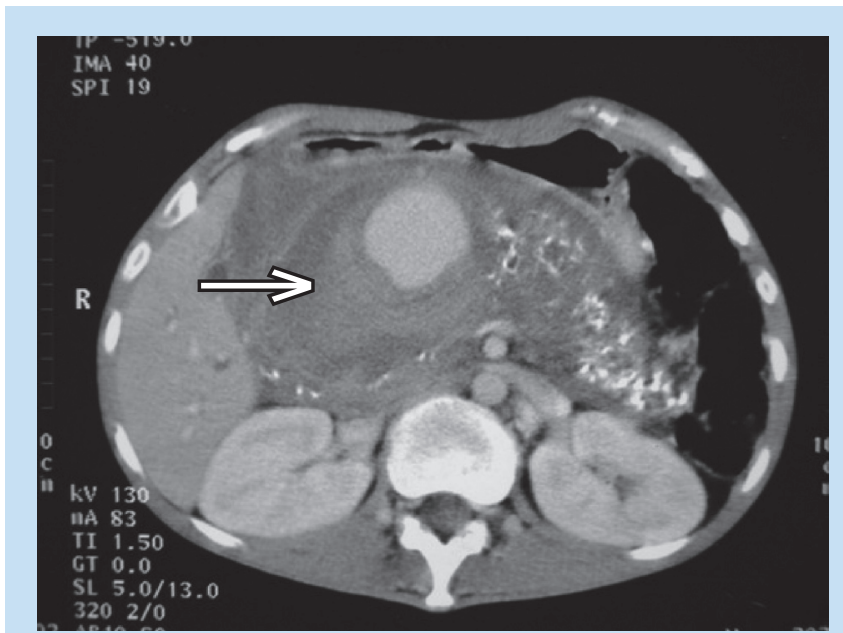

Fig. 1. Axial scan CT of upper abdomen demonstrating expansive mass in the pancreatic head (arrows) and extensive calcifications in pancreatic body and tail.

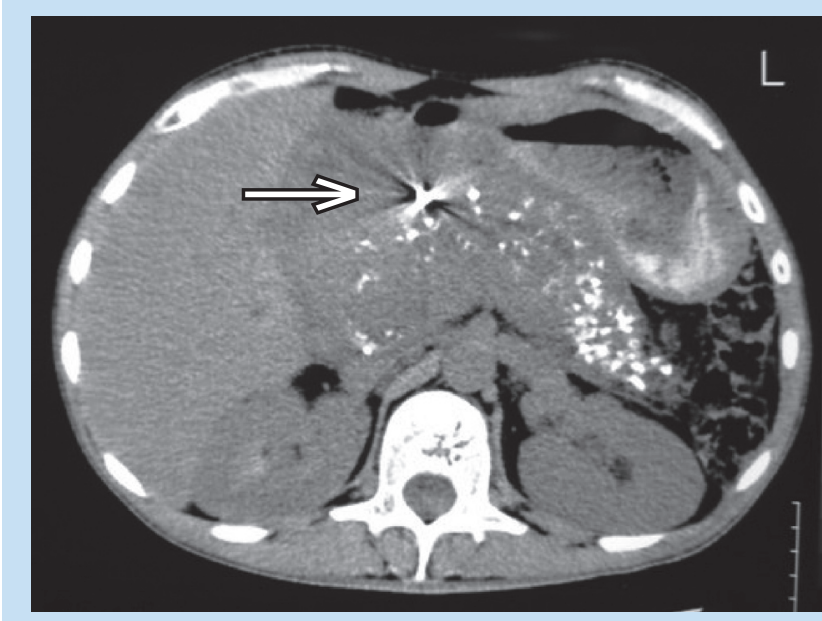

Fig. 2. Common hepatic artery angiography showing gastroduodena artery pseudoaneurysm (arrow).

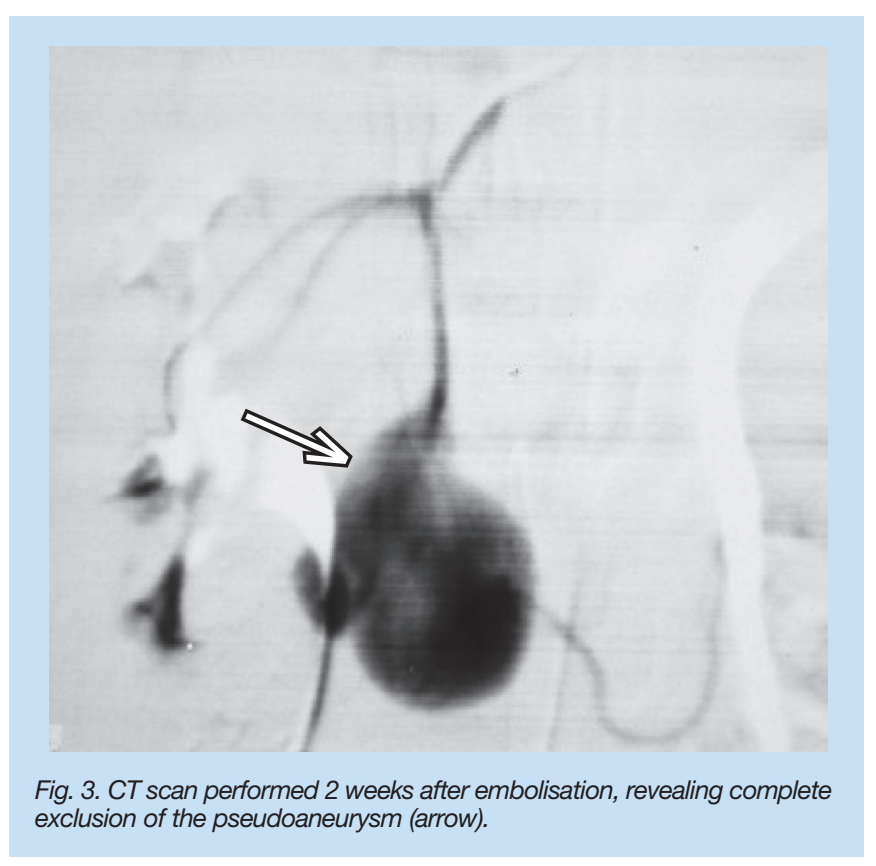

On admission, mild anaemia (haemoglobin $9.3 \mathrm{~g} / \mathrm{dl}$ ), slight hyperglycaemia and mild cholestasis were found. During hospitalisation, the patient was haemodynamically stable but experienced a further drop in haemoglobin level to $7.6 \mathrm{~g} / \mathrm{dl}$. To clarify the cause of his upper gastrointestinal bleeding, an oesophagogastroduodenoscopy was performed, which showed normal endoscopic findings.

A plain and contrast-enhanced abdominal computed tomography (CT) scan revealed multiple pancreatic body calcifications and a dilated biliary tree. In the region of the pancreatic head, a $6 \mathrm{~cm}$ pseudoaneurysm with contained contrast enhancement was identified (Fig. 1). 
These CT findings supported the possibility of secondary haemorrhage into a pancreatic head pseudocyst. Diagnostic angiography with selective common hepatic artery and superselective gastroduodenal artery angiograms showed extravasation of contrast medium into a $4-5 \mathrm{~cm}$ pseudoaneurysm arising from the proximal third of the gastroduodenal artery, approximately $4 \mathrm{~cm}$ from the origin. A catheter was placed deep into the gastroduodenal artery inferior to the point of extravasation, and embolisation with microspheres followed by Gianturco coils was performed.

A post-embolisation angiogram confirmed successful complete occlusion of the gastroduodenal artery and absence of blood flow in the pseudoaneurysm (Fig. 2). The immediate post-embolisation period passed without any complications. The pain subsided and the patient was discharged the following day.

A CT scan performed 2 weeks post-embolisation revealed complete occlusion of the pseudoaneurysm with a significant decrease of biliary tree dilatation (Fig. 3). Over the following 6 months, the patient was re-admitted twice with recurrence of pain secondary to chronic pancreatitis, but no further haemorrhage.

\section{Discussion}

Peri-pancreatic pseudoaneurysms in patients with chronic pancreatitis are an uncommon but serious complication. Mortality rates can reach $40 \%$, depending on the clinical status, site and characteristics of the bleeding lesion, and the surgical management. ${ }^{1}$ Arterial lesions related to pancreatitis are predominantly localised in the splenic artery, the pancreaticoduodenal arteries and the gastroduodenal artery.

Although gastroduodenal artery pseudoaneurysms develop mainly secondarily to pancreatitis, other rare causes such as atherosclerosis, trauma, iatrogenic lesions, tuberculous inflammation and connective tissue diseases have been reported.

The pathogenesis of pseudoaneurysms in pancreatitis results from the proteolytic action of the pancreatic enzymes in the pseudocyst (mainly the elastase) in direct contact with the visceral artery, thereby converting the pseudocyst into a large pseudoaneurysm. The symptoms of such post-pancreatitis pseudoaneurysms may be masked by the clinical features of chronic pancreatitis, until they rupture into the gastrointestinal tract, biliary tree, peritoneal cavity or retroperitoneum, manifesting with the dramatic clinical symptoms of haemorrhagic shock and consequently high mortality rates. ${ }^{2}$ In chronic pancreatitis, several factors may increase the risk of acute bleeding, such as duration of disease, proximity of the vessel to a pseudocyst, communication with the biliary or pancreatic ducts, and splenic vein occlusion due to thrombosis.
In the diagnostic workup, non-invasive diagnostic procedures are the priority: ultrasonography with colour Doppler, endoscopic ultrasonography and contrast-enhanced CT, including CT angiography. The pseudoaneurysm size, presence of thrombus, and pseudoaneurysm topography can often be well defined. Catheter angiography remains important in the visualisation and measuring of the exact gastroduodenal artery pathology and artery width, and presence of arteriovenous fistula. It is an invasive procedure, but can also allow treatment of the pseudoaneurysm at the same time. ${ }^{2}$

In cases of haemodynamic instability, patients have in the past undergone urgent surgery. The optimal treatment of pseudoaneurysms in chronic pancreatitis with haemodynamic stability remains controversial, and there are as yet no evidence-based guidelines, owing to limited data. In our opinion, the risks and difficulties of major surgical intervention made embolisation preferable.

Recent studies have confirmed the effectiveness of arteriographic embolisation or ultrasound-guided thrombin injection ${ }^{3}$ for temporary and definitive control of bleeding from pseudoaneurysms associated with chronic pancreatitis. ${ }^{3}$ However, some authors consider surgery as the treatment of choice for bleeding pseudoaneurysms in patients with chronic pancreatitis, arguing that embolisation does not cure the diseased pancreas and that subsequent surgery is always indicated, anyway. Also, Jun-Te $\mathrm{Hsu}^{4}$ found in his study that embolisation had a high overall treatment failure rate of $20 \%$ and a mortality rate of $20 \%$. As chronic pancreatitis is an ongoing inflammatory process, it is accepted that embolisation may be only a temporary measure, followed by operative intervention as required.

Embolisation failure is an indication for operative treatment, although there is debate about the best surgical procedure. Some authors suggest that proximal and transcystic ligation of bleeding vessels with internal or external cyst drainage is superior to pancreatic resection in the acute situation, although resection is acknowledged to prevent bleeding recurrence in chronic pancreatitis and should be employed when possible.

\footnotetext{
1. Balachandra S, Siriwardena AK. Systemic appraisal of the management of the major vascular complications of pancreatitis. Am J Surg 2005; 190: 489-495.

2. Parildar M, Oran I, Memis A. Embolization of visceral pseudoaneurysms with platinum coils and N-butyl cyanoacrylate. Abdom Imaging 2003; 28: 36-40.

3. Bergert H, Hinterseher I, Kersting S, Leonhardt I, Bloomenthal A, Saeger HD. Management and outcome of hemorrhage due to arterial pseudoaneurysms in pancreatitis. Surgery 2005; 137: 323-328.

4. Jun-Te Hsu, Chun-Nan Yeh, Chien-Fu Hung, et al. Management and outcome of bleeding pseudoaneurysm associated with chronic pancreatitis. BMC Gastroenterol 2006; 6: 3 .
} 